Japan. J. Math.

Vol. 19, No. 2, 1994

\title{
On real quadratic fields with a single class in each genus
}

\author{
By Kazuhiro Dohmae
}

(Received May 19, 1992)

\section{§1. Introduction}

Let $D$ be a square-free rational integer and $d$ the discriminant of the quadratic field $K=\mathbf{Q}(\sqrt{D})$. We denote by $H^{+}(K)$ (resp. $H(K)$ ) the ideal class group of $K$ in the narrow sense (resp. in the wide sense). If $\#\left(H^{+}(K)^{2}\right)=1$, we say that the quadratic field $K$ has a single class in each genus. It is easy to see that $\#\left(H^{+}(K)^{2}\right)=$ 1 implies $\#\left(H(K)^{2}\right)=1$. The ideal class group $H(K)$ of $K$ is said to be trivial if $\#\left(H(K)^{2}\right)=1$ (i.e., if $H(K)$ is generated by the classes of order 2).

In this paper, we shall study real quadratic fields with a single class in each genus. In particular, we shall show that there are at most seventy real quadratic fields of narrow $\mathrm{R}-\mathrm{D}$ type which have a single class in each genus, and determine sixty-nine of them completely (Theorem 2 in section 4 ). To get this theorem, we use Tatuzawa's lower bound for $L(1, \chi)$ (Lemma 1 in section 2 ) and some necessary condition of the solvability of the diophantine equation $x^{2}-D y^{2}= \pm 4 m$ (Lemma 3 in section 3). In [8], P. J. Weinberger studied imaginary quadratic fields with a single class in each genus. He showed that there are at most sixty-eight imaginary quadratic fields having a single class in each genus by using Tatuzawa's lower bound. (It is still an open problem whether there exists this sixty-eighth one.)

In section 2 , we give an upper bound of discriminants of a certain kind of real quadratic fields with a single class in each genus. Applying this result, if the real quadratic field $\mathbf{Q}(\sqrt{D})$ of $\mathrm{R}-\mathrm{D}$ type has a single class in each genus, we obtain $D \leq 1.31 \times 10^{16}$ with one possible exception. Section 3 is devoted to the study of the triviality of ideal class groups. In [6], using the theory of continued fractional expansions, S. Louboutin studied real quadratic fields whose ideal class groups are trivial. But we study the triviality in terms of the solvability of the diophantine equations. In section 4 , we prove the following theorem:

Theorem 2. Let $D$ be of narrow $R-D$ type. Then we have:

(i) There are 69 values of $D$ such that $D \leq 1.31 \times 10^{16}$ and $\mathbf{Q}(\sqrt{D})$ has a single class in each genus.

(ii) There is at most one $D$ such that $D>1.31 \times 10^{16}$ and $\mathbf{Q}(\sqrt{D})$ has a single class in each genus. 


\section{§2. The estimation of discriminants}

In this section, we shall majorize the discriminants of real quadratic fields which have a single class in each genus. Let $D$ be a square-free positive rational integer $(>1)$ and $d$ the discriminant of the real quadratic field $K=\mathbf{Q}(\sqrt{D})=\mathbf{Q}(\sqrt{d})$. We denote the ideal class number of $K$ (in the narrow sense) by $h^{+}(K)$, and the genus number of $K$ by $g^{+}(K)$. Let $\chi_{d}$ be the non-principal primitive real Dirichlet character modulo $d$, and $L\left(s, \chi_{d}\right)$ the corresponding L-function. Furthermore, we denote by $\epsilon_{d}$ or $\epsilon_{D}$ the fundamental unit of $K$ which is greater than one. The real quadratic field $K$ has a single class in each genus if and only if $h^{+}(K)=g^{+}(K)$.

The following lemma is essential for our purpose:

Lemma 1 ([7]). Let $0<\varepsilon<1 / 2$ and $d \geq \max \left(e^{1 / \varepsilon}, e^{11.2}\right)$. Then

$$
L\left(1, \chi_{d}\right)>0.655 \frac{\varepsilon}{d^{\varepsilon}}
$$

with one possible exception.

REMARK 1. If GRH holds, we can remove the possible exception.

We put $d_{k}=p_{1} p_{2} \ldots p_{k}$, where $p_{n}$ is the $n$-th prime.

Proposition 1. For $C \geq 1$ and $\alpha \geq 1 / 2$, we set $\Delta=\left\{d \mid \epsilon_{d} \leq C d^{\alpha}\right\}$. Let $k$ be the least positive rational integer such that

(i) $\quad p_{k+1}{ }^{\beta} \geq 6 \quad\left(\beta=\frac{1}{2}-\frac{1}{\log d_{k}}\right)$,

(ii) $\frac{0.655}{2 e} \frac{\sqrt{d_{k}}}{\log d_{k} \cdot\left(\log C+\alpha \log d_{k}\right)}>2^{k-1}$.

If $d \in \Delta$ and $d \geq d_{k}$, then

$$
h^{+}(\mathbf{Q}(\sqrt{d}))>g^{+}(\mathbf{Q}(\sqrt{d}))
$$

with one possible exception.

REMARK 2. (i) It follows from $p_{k+1}{ }^{\beta} \geq 6$ that $k$ is greater than or equal to 14.

(ii) We can always find a positive rational integer $k$ which satisfies the condition of Proposition 1, because

$$
\lim _{k \rightarrow \infty} \frac{\sqrt{d_{k}}}{2^{k-1} \log d_{k} \cdot\left(\log C+\alpha \log d_{k}\right)}=+\infty .
$$

REMARK 3. Changing the definition of the set $\Delta$, we can make up other estimating formulas easily. 
Proof. Suppose that $d \in \Delta, d \geq d_{k}$ and $d$ is the product of $t$ prime discriminants. Let $\varepsilon=1 / \log d_{k}$ in Lemma 1 , and $d$ not be the possible exception. Then, by the analytic class number formula,

$$
\begin{aligned}
h^{+}(\mathbf{Q}(\sqrt{d})) & \geq \frac{\sqrt{d}}{2 \log \epsilon_{d}} L\left(1, \chi_{d}\right) \\
& >\frac{\sqrt{d}}{2 \log \left(C d^{\alpha}\right)} \cdot 0.655 \frac{1}{\log d_{k} \cdot d^{1 / \log d_{k}}} \\
& =\frac{0.655}{2 \log d_{k}} \cdot \frac{d^{\beta}}{\log C+\alpha \log d} .
\end{aligned}
$$

Since the last term above is an increasing function of $d$, from the condition (ii), we get

$$
h^{+}(\mathbf{Q}(\sqrt{d}))>\frac{0.655}{2 e} \cdot \frac{\sqrt{d_{k}}}{\log d_{k} \cdot\left(\log C+\alpha \log d_{k}\right)}>2^{k-1} .
$$

Now we suppose that $h^{+}(\mathbf{Q}(\sqrt{d}))=g^{+}(\mathbf{Q}(\sqrt{d}))$. Then

$$
2^{t-1}=g^{+}(\mathbf{Q}(\sqrt{d}))=h^{+}(\mathbf{Q}(\sqrt{d}))>2^{k-1},
$$

so $t>k$. Hence $d \geq d_{k} p_{k+1}{ }^{t-k}$, and we obtain

$$
\begin{aligned}
2^{t-1} & =h^{+}(\mathbf{Q}(\sqrt{d})) \\
& >\frac{0.655}{2 \log d_{k}} \cdot \frac{d_{k}{ }^{\beta} p_{k+1}{ }^{\beta(t-k)}}{\left(\log C+\alpha \log d_{k}+\alpha(t-k) \log p_{k+1}\right)} \\
& \geq \frac{0.655}{2 e} \cdot \frac{\sqrt{d_{k}} \cdot 6^{t-k}}{\log d_{k} \cdot\left(\log C+\alpha \log d_{k}+\alpha(t-k) \log p_{k+1}\right)}
\end{aligned}
$$

by using the inequality (3) and the condition (i). Therefore

$$
\begin{aligned}
2^{k-1} & >\frac{0.655}{2 e} \frac{\sqrt{d_{k}} \cdot 3^{t-k}}{\log d_{k} \cdot\left(\log C+\alpha \log d_{k}+\alpha(t-k) \log p_{k+1}\right)} \\
& \geq \frac{0.655}{2 e} \cdot \frac{\sqrt{d_{k}}}{\log d_{k} \cdot\left(\log C+\alpha \log d_{k}\right)}>2^{k-1}
\end{aligned}
$$

because the second term is an increasing function of $t$. This is a contradiction. Hence

$$
h^{+}(\mathbf{Q}(\sqrt{d}))>g^{+}(\mathbf{Q}(\sqrt{d})) .
$$

Corollary 1. We put $\epsilon_{D}=\left(t_{D}+u_{D} \sqrt{D}\right) / 2(>1)$. For any fixed positive rational integer $u$, the number of real quadratic fields $\mathbf{Q}(\sqrt{D})$ such that $h^{+}(\mathbf{Q}(\sqrt{D}))=$ $g^{+}(\mathbf{Q}(\sqrt{D}))$ and $u_{D}=u$ is finite. 
Proof. . We can easily see that $\epsilon_{D} \leq\left(u_{D}+1\right) \sqrt{D}$. Then the result follows from Proposition 1.

A real quadratic field $\mathbf{Q}(\sqrt{D})$ (or simply $D$ ) is said to be of R-D type if $D=n^{2}+r \neq 5$ where $-n<r \leq n$ and $r \mid 4 n$. If $r \in\{ \pm 1, \pm 4\}$, then $D$ is said to be of narrow $\mathrm{R}-\mathrm{D}$ type. The fundamental units of real quadratic fieds of $\mathrm{R}-\mathrm{D}$ type are explicitly known:

$$
\epsilon_{D}= \begin{cases}n+\sqrt{D} & \text { if } \quad r= \pm 1, \\ \frac{n+\sqrt{D}}{2} & \text { if } r= \pm 4 \\ \frac{2 n^{2}+r+2 n \sqrt{D}}{|r|} & \text { if } \quad r \neq \pm 1, \pm 4 .\end{cases}
$$

If $D$ is of R-D type, it is easy to see that $\epsilon_{D} \leq 4 D$. Hence, we obtain:

Corollary 2. Let $D$ be of $R-D$ type. If $h^{+}(\mathbf{Q}(\sqrt{D}))=g^{+}(\mathbf{Q}(\sqrt{D}))$, then

$$
D \leq d_{14}\left(<1.31 \times 10^{16}\right)
$$

with one possible exception.

\section{§3. Triviality of ideal class groups}

Let $D$ be a square-free positive rational integer. We denote by $H(D)$ and $\epsilon_{D}$ the ideal class group (in the wide sense) and the fundamental unit $(>1)$ of the real quadratic field $\mathbf{Q}(\sqrt{D})$. We put

$$
\left(\frac{D}{p}\right)^{*}=\left\{\begin{array}{lll}
\left(\frac{D}{p}\right) & \cdots & p \text { odd prime } \\
1 & \cdots & p=2 \text { and } D \equiv 1(\bmod 8) \\
-1 & \cdots & p=2 \text { and } D \equiv 5(\bmod 8) \\
0 & \cdots & p=2 \text { and } D \equiv 2 \text { or } 3(\bmod 4)
\end{array}\right.
$$

Let $d$ be the discriminant of the real quadratic field $\mathbf{Q}(\sqrt{D})$, and $\chi_{d}$ the nonprincipal primitive real Dirichlet character modulo $d$. Then we have $\chi_{d}(p)=\left(\frac{D}{p}\right)^{*}$ for any prime $p$. A prime $p$ splits completely in $\mathbf{Q}(\sqrt{D})$ if and only if $\left(\frac{D}{p}\right)^{*}=1$.

The ideal class group $H(D)$ of $\mathbf{Q}(\sqrt{D})$ is said to be trivial if it is generated by the classes of order $2 . H(D)$ is trivial if and only if $\#\left(H(D)^{2}\right)=1$. We consider the diophantine equation

$$
x^{2}-D y^{2}= \pm 4 m
$$


for any positive rational integer $m(>1)$. Let $\left(x_{0}, y_{0}\right)$ be an integral solution of the diophantine equation $(*)$. If $m=n^{2}$ with some rational integer $n$ and if $x_{0} \equiv y_{0} \equiv$ $0(\bmod n)$, then we say that the integral solution $\left(x_{0}, y_{0}\right)$ is trivial.

In this section, we shall give some conditions for the triviality of the ideal class group $H(D)$ in terms of the solvability of the diophantine equation $(*)$. First, we prove the following lemma:

Lemma 2 (cf. [10]). Let $p$ be a prime. Then the following two statements are equivalent:

(i) The diophantine equation $x^{2}-D y^{2}= \pm 4 p^{2}$ has a non-trivial integral solution.

(ii) The prime $p$ splits in $K=\mathbf{Q}(\sqrt{D})$ as $(p)=\mathfrak{p p}^{\prime}$, where $\mathfrak{p} \neq \mathfrak{p}^{\prime}$ and $\mathfrak{p}^{2}$ is principal.

Proof. (i) $\Rightarrow$ (ii) Let $\left(x_{0}, y_{0}\right)$ be a non-trivial integral solution of the diophantine equation $x^{2}-D y^{2}= \pm 4 p^{2}$. Then

$$
\pm p^{2}=\frac{x_{0}^{2}-D y_{0}^{2}}{4}=\frac{x_{0}+y_{0} \sqrt{D}}{2} \frac{x_{0}-y_{0} \sqrt{D}}{2}
$$

so

$$
(p)^{2}=(\alpha)(\alpha)^{\prime}
$$

where $\alpha=\left(x_{0}+y_{0} \sqrt{D}\right) / 2$ and $(\alpha) \neq(\alpha)^{\prime}$ since $p \nmid x_{0} y_{0}$. If $p \geq 3$, then

$$
\left(\frac{D}{p}\right)^{*}=\left(\frac{D}{p}\right)=\left(\frac{D y_{0}^{2}}{p}\right)=\left(\frac{x_{0}^{2}}{p}\right)=1 \text {. }
$$

If $p=2$, then $x_{0}^{2} \equiv y_{0}^{2} \equiv 1(\bmod 8)$, consequently

$$
\left(\frac{D}{2}\right)^{*}=1
$$

Hence, $p$ splits completely in $\mathrm{K}$ :

$$
(p)=\mathfrak{p p}^{\prime}, \quad \mathfrak{p} \neq \mathfrak{p}^{\prime}
$$

From (1) and (2), we obtain

$$
\mathfrak{p}^{2} \mathfrak{p}^{\prime 2}=(\alpha)(\alpha)^{\prime}
$$

Therefore, $\mathfrak{p}^{2}=(\alpha)$ or $(\alpha)^{\prime}$, so $\mathfrak{p}^{2}$ is principal.

(ii) $\Rightarrow$ (i) We put

$$
\mathfrak{p}^{2}=(\alpha), \quad \alpha=\frac{x_{0}+y_{0} \sqrt{D}}{2}
$$


Then

$$
p^{2}=\mathrm{N}_{K / Q} \mathfrak{p}^{2}=\mathrm{N}_{K / Q}(\alpha)=\left|\mathrm{N}_{K / Q} \alpha\right|=\left|\frac{x_{0}^{2}-D y_{0}^{2}}{4}\right|
$$

so

$$
x_{0}^{2}-D y_{0}^{2}= \pm 4 p^{2} \text {. }
$$

Since $(p)=\mathfrak{p p}^{\prime} \nmid \mathfrak{p}^{2}=(\alpha)$, we have $x_{0} \not \equiv 0(\bmod p)$ and $y_{0} \not \equiv 0(\bmod p)$. Hence $\left(x_{0}, y_{0}\right)$ is a non-trivial integral solution of the diophantine equation $x^{2}-D y^{2}=$ $\pm 4 p^{2}$.

We denote by $M_{D}$ the Minkowski constant of the real quadratic field $\mathbf{Q}(\sqrt{D})$ :

$$
M_{D}=\left\{\begin{array}{ccl}
\frac{\sqrt{D}}{2} & \cdots & D \equiv 1(\bmod 4) \\
\sqrt{D} & \cdots & D \equiv 2 \text { or } 3(\bmod 4) .
\end{array}\right.
$$

In any ideal class of $\mathbf{Q}(\sqrt{D})$, there exists an (integral) ideal $I$ such that $\mathrm{N}_{K / \mathrm{Q}} I \leq$ $M_{D}$. Moreover, if we put

$$
S_{D}=\left\{p \mid p \text { prime, } p \leq M_{D} \text { and }\left(\frac{D}{p}\right)^{*}=1\right\},
$$

then we obtain the following theorem by using Lemma 2 .

THEOREM 1. The following statements are equivalent:

(i) The ideal class group $H(D)$ of $\mathbf{Q}(\sqrt{D})$ is trivial (i.e., $\left.\#\left(H(D)^{2}\right)=1\right)$.

(ii) For any prime $p \in S_{D}$, the diophantine equation $x^{2}-D y^{2}= \pm 4 p^{2}$ has a non-trivial integral solution.

(iii) For any prime $p$ such that $\left(\frac{D}{p}\right)^{*}=1$, the diophantine equation $x^{2}-$ $D y^{2}= \pm 4 p^{2}$ has a non-trivial integral solution.

Proof. Straightforward.

This theorem gives a necessary and sufficient condition for the triviality of the ideal class group of $\mathbf{Q}(\sqrt{D})$. But it is not so easy to see whether the diophantine equation $x^{2}-D y^{2}= \pm 4 p^{2}$ has a non-trivial integral solution. So, by using this theorem and the following lemma, we deduce some necessary conditions which are more useful for us.

LEMMA 3 ([10]). Let $m>1$ be a rational integer, and decide $t_{D}$ and $u_{D}$ by

$$
\epsilon_{D}=\frac{t_{D}+u_{D} \sqrt{D}}{2}
$$


If the diophantine equation $x^{2}-D y^{2}= \pm 4 m$ has a non-trivial integral solution, the following inequality holds:

$$
m \geq s_{D}=\left\{\begin{array}{lll}
\frac{t_{D}}{u_{D}{ }^{2}} & \cdots & N \epsilon_{D}=-1 \\
\frac{t_{D}-2}{u_{D}{ }^{2}} & \cdots & N \epsilon_{D}=+1
\end{array}\right.
$$

REMARK 4. For real quadratic fields of narrow R-D type, we have:

(i) If $D=a^{2}+4$ or $4 a^{2}+1$, then $s_{D}=a$.

(ii) If $D=a^{2}+1 \equiv 2(\bmod 4)$, then $s_{D}=a / 2$.

(iii) If $D=a^{2}-4$, then $s_{D}=a-2$.

(iv) If $D=4 a^{2}-1$, then $s_{D}=a-1 / 2$.

For $s_{D}$ which is defined in Lemma 3 , we put $r_{D}=\sqrt{s_{D}}$.

Proposition 2. Let $D$ be of narrow $R-D$ type. If there exists a prime $p$ such that $p<r_{D}$ and $\left(\frac{D}{p}\right)^{*}=1$, then $\#\left(H(D)^{2}\right)>1$.

Proof. Since $p<r_{D} \leq M_{D}$ and $\left(\frac{D}{p}\right)^{*}=1$, we get $p \in S_{D}$. Suppose that $\#\left(H(D)^{2}\right)=1$. Then, by Theorem 1 , the diophantine equation $x^{2}-D y^{2}= \pm 4 p^{2}$ has a non-trivial integral solution. This contradicts Lemma 3.

Proposition 3. (i) If $D=a^{2}+4$ and if $\#\left(H(D)^{2}\right)=1$, then we have

$$
a=1, p \text { or } p^{2}
$$

where $p$ is an odd prime.

(ii) If $D=4 a^{2}+1$ and if $\#\left(H(D)^{2}\right)=1$, then we have

$$
a=1, p \text { or } p^{2}
$$

where $p$ is a prime.

(iii) If $D=a^{2}+1 \equiv 2(\bmod 4)$ and if $\#\left(H(D)^{2}\right)=1$, then we have

$$
a=1, p \text { or } p q
$$

where $p$ and $q$ are odd primes such that $p \leq q \leq 2 p$.

Proof. The statements (i), (ii) and (iii) can be proved in the same way. Here we prove only (i). We suppose that $a \neq 1$. Let $p$ be the least prime factor of $a$. We put

$$
a=p n \quad(n=1 \text { or } n \geq p)
$$


The prime $p$ splits completely in $\mathbf{Q}(\sqrt{D})$, because $D \equiv 4=2^{2}(\bmod p)$. So, from Theorem 1, the diophantine equation $x^{2}-D y^{2}= \pm 4 p^{2}$ has a non-trivial integral solution. Hence, by using Lemma 3 , we get

$$
p^{2} \geq a=p n
$$

then we have $n \leq p$. Therefore we obtain $a=p$ or $p^{2}$.

Remark 5. Propositions 2 and 3 are elementary, but they are decisive conditions for actual computation. Because, for the real quadratic field $\mathbf{Q}(\sqrt{D})$ of narrow $\mathrm{R}-\mathrm{D}$ type, we have $r_{D}=\mathrm{O}\left(D^{\frac{1}{4}}\right)$. It is well-known that the least prime quadratic residue modulo $d$ is $\mathrm{O}\left(d^{\frac{1}{4}+\varepsilon}\right)$ for any $\varepsilon>0$. (cf. [4])

\section{§4. Main result and table}

Now we can determine the real quadratic fields of narrow R-D type (except for at most one) which have a single class in each genus.

First, using pascal on Sun Microsystems, we pick up all $D$ 's which are less than or equal to $1.31 \times 10^{16}$ and satisfy at least one of the following conditions:

(i) $D=a^{2}+4$, where $a=1, p$ or $p^{2}$ ( $p$ odd prime), and $\left(\frac{D}{q}\right)^{*} \neq 1$ for any prime $q<\sqrt{a}$,

(ii) $D=4 a^{2}+1$, where $a=1, p$ or $p^{2}$ ( $p$ prime), and $\left(\frac{D}{q}\right)^{*} \neq 1$ for any prime $q<\sqrt{a}$,

(iii) $D=a^{2}+1 \equiv 2(\bmod 4)$, where $a=1, p_{1}$ or $p_{1} p_{2}\left(p_{1}, p_{2}\right.$ are odd primes; $p_{1} \leq p_{2} \leq 2 p_{1}$ ), and $\left(\frac{D}{q}\right)^{*} \neq 1$ for any prime $q<\sqrt{a / 2}$,

(iv) $D=a^{2}-4$, where $a$ is a positive rational integer, and $\left(\frac{D}{q}\right)^{*} \neq 1$ for any prime $q<\sqrt{a-2}$,

(v) $D=4 a^{2}-1$, where $a$ is a positive rational integer, and $\left(\frac{D}{q}\right)^{*} \neq 1$ for any prime $q<\sqrt{a-1 / 2}$.

Then, we compute their ideal class numbers by using UBASIC86 Ver.8.12 on NEC PC-9801RX. Finally, we obtain the following theorem:

Theorem 2. Let $D$ be of narrow $R-D$ type. Then we have:

(i) There are 69 values of $D$ (as in Table) such that $D \leq 1.31 \times 10^{16}$ and $\mathbf{Q}(\sqrt{D})$ has a single class in each genus.

(ii) There is at most one $D$ such that $D>1.31 \times 10^{16}$ and $\mathbf{Q}(\sqrt{D})$ has a single class in each genus. 
Table: $D$ 's of narrow R-D type with a single class in each genus

$$
\left(D \leq 1.31 \times 10^{16}\right)
$$

\begin{tabular}{|c|c|c|c|c|}
\hline$h^{+}(\mathbf{Q}(\sqrt{D}))$ & $D=a^{2}+4$ & $D=a^{2}+1$ & $D=a^{2}-4$ & $D=a^{2}-1$ \\
\hline 1 & $\begin{array}{c}29,53 \\
173,293\end{array}$ & $\begin{array}{c}2,17, \\
37,101, \\
197,677\end{array}$ & & \\
\hline 2 & $\begin{array}{c}85,365 \\
533,629 \\
965,1685 \\
1853,2813\end{array}$ & $\begin{array}{c}10,26, \\
65,122, \\
362,485, \\
1157,2117, \\
3365\end{array}$ & $\begin{array}{c}21,77 \\
437\end{array}$ & 3 \\
\hline 4 & $\begin{array}{c}2405,3485, \\
10205, \\
16133\end{array}$ & $\begin{array}{c}170,290, \\
530,962, \\
1370,9605, \\
14885 \\
20165\end{array}$ & $\begin{array}{c}165,285, \\
357,957, \\
1085,2397\end{array}$ & $\begin{array}{c}15,35 \\
143\end{array}$ \\
\hline 8 & 32045 & $\begin{array}{c}2210,5330 \\
58565 \\
77285\end{array}$ & $\begin{array}{c}1365,2805, \\
4485,7917, \\
\quad 8645\end{array}$ & $\begin{array}{l}195,255 \\
483,1295\end{array}$ \\
\hline 16 & & & 26565 & 1155,3135 \\
\hline
\end{tabular}

REMARK 6. $\mathbf{Q}(\sqrt{5})$ and $\mathbf{Q}(\sqrt{13})$ also have a single class in each genus. But they are not of R-D type.

REMARK 7. To determine the real quadratic fields of non-narrow R-D type which have a single class in each genus, we have to find another method. Because, for a square-free positive rational integer $D$ of non-narrow $\mathrm{R}-\mathrm{D}$ type, the constant $s_{D}($ defined in Lemma 3$)$ is too small.

\section{References}

[ 1 ] S. Chowla and W. E. Briggs, On discriminants of binary quadratic forms with a single class in each genus, Canad. J. Math., 6 (1954), 463-470.

[ 2 ] L. E. Dickson, Introduction to the theory of numbers, University of Chicago (1929). 
[ 3 ] H. K. Kim, M. G. Leu and T. Ono, On two conjectures on real quadratic fields, Proc. Japan Acad. Ser.A Math. Sci., 63 (1987), 222-224.

[ 4 ] Ju. V. Linnik, Sur une application du théorèm d'André Weil à la théorie des caractères de Dirichlet, Séminaire Delange-Pisot-Poitou (Théorie des nombres) 8e année, 1966/67.

[ 5 ] S. Louboutin, R. A. Mollin and H. C. Williams, Class numbers of real quadratic fields, continued fractions, reduced ideals, prime-producing quadratic polynomials and quadratic residue covers (preprint).

[ 6 ] S. Louboutin, Groupes des classes d'idéaux triviaux, Acta. Arith., 54 (1989), 61-74.

[ 7 ] T. Tatuzawa, On a theorem of Siegel, Japan. J. Math., 21 (1951), 163-178.

[ 8 ] P. J. Weinberger, Exponents of the class groups of complex quadratic fields, Acta. Arith., 22 (1973), 117-124.

[ 9 ] H. Yokoi, Class number one problem for real quadratic fields (the conjecture of Gauss), Proc. Japan Acad. Ser.A Math. Sci., 64 (1988), 53-55.

[10] H. Yokoi, Some relations among new invariants of prime number $p$ congruent to $1 \bmod 4$, Adv. Stud. Pure Math., 13 (1988), 493-501.

[11] H. Yokoi, Class-number one problem for certain kind of real quadratic fields, Proc. Int. Conf. on Class Numbers and Fundamental Units, Katata, Japan (1986), 125-137.

DEPARTMENT OF MATHEMATICS TOKYO METROPOLITAN UNIVERSITY 